\title{
Operative Versus Non-Operative Management of Distal Biceps Brachii Tendon Rupture: A Systematic Review
}

\author{
M. K. Qureshi', A. S. Khaled ${ }^{2}$, A. O. Elgindi', S. Mastan'2, M. Jeyam² \\ 1 North Manchester General Hospital, Manchester, UK \\ 2 Salford Royal Hospital, Manchester, UK
}

\author{
CORRESPONDING AUTHOR: \\ Mobeen Khalid Qureshi \\ North Manchester General Hospital \\ 33 Kendall Road \\ Manchester, England \\ E-mail: mobeen.qureshi1@nhs.net
}

DOI:

10.32098/mltj.04.2020.16

LEVEL OF EVIDENCE: 3A

\begin{abstract}
SUMMARY
Background. Distal biceps brachii tendon ruptures occur rarely accounting for 3\% of all biceps tendon injuries. This systematic review aims to assess the outcomes of both operative and non-operative management of distal biceps brachii tendon rupture for adult patients. The literature has mostly advocated that surgical intervention to be the best management of such injuries however in recent times studies have shown acceptable outcomes of non-operative treatment in those affected and therefore offer an alternative to current practise.

Methods. The NICE Healthcare Databases Advanced Search (HDAS) tool was used to identify articles in the PubMed, Medline, EMBASE, and the Cochrane Central Register of Controlled Trials databases to identify articles relating to distal biceps brachii tendon rupture management. The search strategy was formed by using the set key words. Studies published from 1998-2008 were included. Functional outcome questionnaires and isometric strength tests were used. An inclusion criterion identified articles to be critically appraised. Results. Initially 427 papers were identified with the key words in the search approach from the use of the electronic databases. 294 papers were excluded because they were either duplicates or unrelated to distal rupture of the tendon biceps brachii. It recognized 17 papers and abstracts of which 11 were omitted because they were either in a different language or were case reports.

Conclusions. This review highlights that the current evidence for surgical management of distal biceps brachii tendon rupture is weak. Studies in recent times have mostly focused on the different methods of surgical fixation. We noted that surgical fixation resulted in a large number of complications, which if communicated to the patient may result in them opting for conservative management.

However equally important to highlight is that patients who are middle-aged, with a modest physical demand should be made aware that if treated non-operatively they may likely have good to excellent functional outcome as well as having approximately $50 \%$ strength in the flexed and supinated position of the injured arm, whilst avoiding the potential complications of surgery. This review also highlights that more higher quality evidence is required in future.
\end{abstract}

KEY WORDS

Distal biceps brachii tendon rupture; distal biceps tendon rupture; surgical treatment; conservative treatment; systematic review.

\section{INTRODUCTION}

In 1843 Starks reported the first distal biceps brachii tendon rupture. The first repair of this injury was reported fifty years later in 1897 by Johnson $(1,2)$. Distal biceps brachii tendon ruptures occur rarely accounting for $3 \%$ of all biceps tendon injuries (3), with the majority of ruptures occurring in the tendon of the long head of biceps or more frequently in the short head of biceps. It occurs when the flexed and supinated elbow has an abrupt eccentric load applied and most often involves middle aged men who are active and in their 
$4^{\text {th }}$ to $6^{\text {th }}$ decades of life (4), with incidence reported to be 1.2 per 100,000 per year. Smoking and steroid use have been identified as two independent risk factors (5). The literature typically recommends that operative treatment offers the best outcome, with several retrospective case series concluding that strength is best restored with surgical intervention (6). Operative treatment has a reported complication rate ranging from $23 \%$ to $27 \%(7,8)$. This is due to the close proximity to surrounding structures such as the posterior interosseous nerve, the median nerve, the lateral cutaneous nerve to the distal biceps brachii tendon, to common interosseous artery and the radial recurrent branch of the radial artery (9). There are a range of surgical approaches that have been used: a single anterior incision approach (10, 11), a two incision approach developed by Boyd and Anderson (12), a modified two incision approach known as the "Solento Technique" (13), the distal biceps being attached to the brachialis (14), as well techniques which employ a suture anchor, bone tunnel, interference screws or cortical buttons (15). The most common complication with the anterior approach is that of nerve injury, with the posterior interosseous nerve being injured in $5 \%$ of cases and the lateral antebrachial cutaneous nerve being injured in up to $7 \%$ of cases. In the two incision approach the most common complication is that of heterotopic ossification in $10 \%$ to $15 \%$ of cases (16). There are other reported complications such as re-rupture, infection, persistent pain, reduced range of motion, complex regional pain syndrome $(7,8)$. The risks of operative management lead to the question of whether the risks of surgery are greater than the benefits.

Carroll et al. reported that at the time of one year follow up, there was no loss of flexion and supination in twenty patients when treated conservatively for distal biceps brachii tendon rupture (17). Morrey et al. reported that 3 patients who had been treated conservatively had $31 \%$ flexion strength and $40 \%$ supination strength when compared to normal individuals (18). Baker and Bierwagen compared the injured arm with the contralateral arm when treated conservatively and reported that the average differences in flexion and supination strength of $21 \%$ and $27 \%$ respectively, in addition to reduced endurance (19).

More recently there have been studies comparing operative treatment to non-operative management of distal biceps tendon rupture. Chillemi et al. reported in those who had anatomical repair had superior European Society for Surgery of the Shoulder and the Elbow scores (SECEC), in comparison to those who were treated conservatively (20). Hetsroni et al. concluded that even though surgical repair resulted in higher satisfaction, non-operative management resulted in good to excellent outcomes as well and therefore should be a considered treatment plan given the potential complications of surgery (21). Freeman et al. concludes that nonoperative treatment can produce satisfactory outcomes with modestly reduced strength (4). In their study, Pavelka et al. reported that the loss of supination and flexion strength was not as severe as reported in the literature and that non-operative management was an acceptable alternative (22).

The aim of this systematic review is to assess the outcomes of both operative and non-operative management of distal biceps brachii tendon rupture for adult patients. The literature has mostly advocated that surgical intervention to be the best management of such injuries however in recent times studies have shown acceptable outcomes of non-operative treatment in those affected and therefore offer an alternative to current practise, avoiding the potential serious complications associated with repairing a ruptured distal biceps brachii tendon.

\section{METHODS}

To guide the systematic review the Preferred Reporting Items for Systematic Reviews and Meta-Analyses (PRISMA) guidelines were used and adhered to (23). Studies comparing non-operative management to surgical management of distal biceps brachii tendon rupture as well as studies assessing the effectiveness of non-operative management alone were evaluated against the set inclusion criteria. To formulate the inclusion and exclusion criteria (table I), the PICOT criteria was used (24). This focused the search and reduced ambiguity. Surgical intervention included all types of anatomic re-insertion of the distal biceps brachii tendon to the radial tuberosity or any other area such as the brachialis. No restrictions were placed on the way patients were treated conservatively. Studies that used the Disabilities of the Arm, Shoulder and Hand (DASH) questionnaire, shortened Disabilities of the Arm, Shoulder, and Hand (QuickDASH) questionnaire, Single Assessment Numerical Evaluation (SANE), Biceps Disability Questionnaire (BDQ), Oxford Elbow Score (OES), Mayo Elbow Performance Index (MEPI), Visual Analogue Scale (VAS), Broberg and Morrey Functional Rating Index (BMFI), European Society for Surgery of the Shoulder and the Elbow score (SECEC), the Constant Score (CS), American Shoulder and Elbow Surgeons score (ASES) and modified Neer Scale as a way to measure the functional outcome were eligible. Publication time was limited to the years 1998 and 2018 and to English-language papers.

To search for papers related to distal biceps brachii tendon rupture management, the NICE Healthcare Databases Advanced Search (HDAS) tool was used to define articles in the PubMed, Medline, EMBASE and Cochrane Central 
Table I. Criteria for inclusion and exclusion.

\begin{tabular}{lll}
\hline & Inclusion Criteria & Exclusion Criteria \\
\hline Population & -Age $>16$ & $\begin{array}{l}\text {-Age }<16 \\
\text {-Bilateral distal biceps tendon } \\
\text { brachii rupture }\end{array}$ \\
\hline Intervention & $\begin{array}{l}\text {-Operative management of distal biceps brachii } \\
\text { tendon rupture }\end{array}$ & Not Applicable \\
& -Non-operative management of distal biceps brachii & \\
\hline Comparison Group & -Studies comparing operative with non-operative & Not Applicable \\
& management & \\
\hline Outcome & -Studies measuring an outcome of the intervention using & $\begin{array}{l}\text {-Studies not measuring functional outcome } \\
\text { or isokinetic strength }\end{array}$ \\
\hline Time & standardised scores & -Studies published prior to 1998 \\
\hline Study type & -Studies between the years 1998 to 2018 & -Letters \\
& -Randomised Control Trials & -Case reports \\
\hline Language & - Clinical Trials & \\
\hline & - Cohort Studies & \\
\hline
\end{tabular}

Register of Controlled Trials databases. Two independent co-authors (M.Q, A.E.) undertook the search process. All relevant articles and reviews were screened for suitable papers. To formulate the search approach, the following main terms were used:

- "Distal Biceps Tendon Rupture" OR "Distal biceps brachii rupture" AND "Non-operative treatment" OR "Non-operative management" OR "Conservative management" OR "Conservative treatment".

- "Distal Biceps Tendon Rupture" OR "Distal biceps brachii rupture" AND "Operative treatment" OR "Operative management" OR "Surgical management" OR "Surgical treatment".

- "Distal Biceps Tendon Rupture" OR "Distal biceps brachii rupture" AND "Non-operative management versus operative management" OR "Non-operative treatment versus operative treatment" OR "Conservative management versus surgical management" OR "Conservative treatment versus surgical treatment".

Articles that matched all elements of the requirements for inclusion were then critically evaluated using the Critical Appraisal Skills Program (CASP) tool (25) and The Cochrane Handbook for Systematic Reviews of Interventions (26) (figure 1).

This review meets the ethical standards of the Muscles, Ligaments and Tendon Journal (27).

\section{RESULTS}

Initially 427 papers were identified with the key words used in the search approach from the use of the electronic databases. 294 papers were excluded because they were either duplicates or unrelated to distal rupture of the tendon biceps

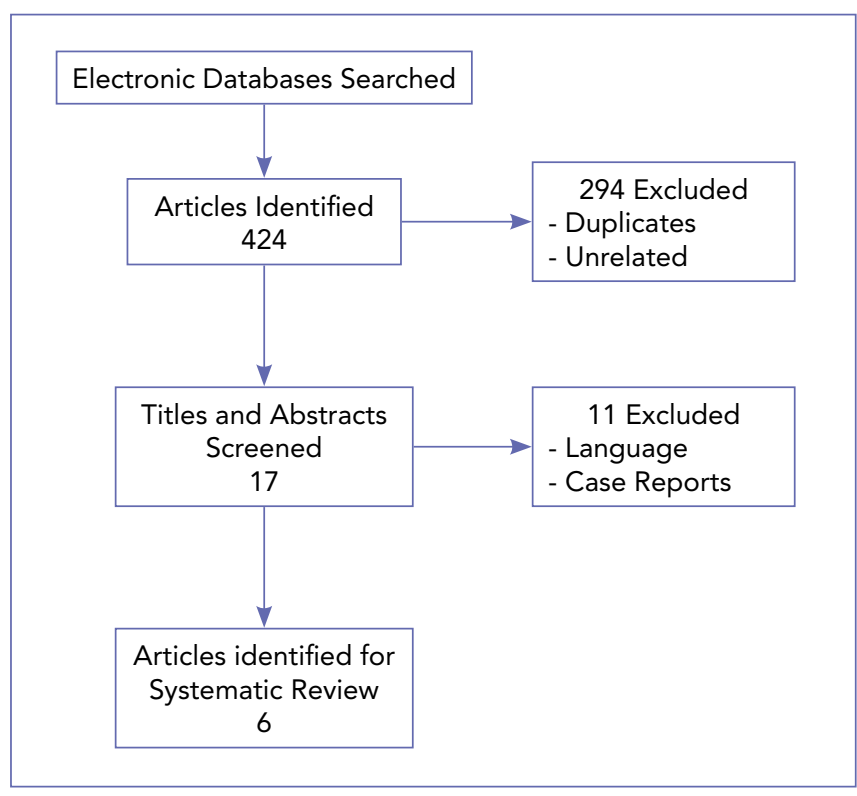

Figure 1. Flow diagram of search process.

Muscles, Ligaments and Tendons Journal 2020;10 (4) 
brachii. It recognized 17 papers and abstracts of which 11 were omitted because 3 were in a different language and 8 were case reports.

\section{DISCUSSION}

Surgical treatment of distal biceps brachii tendon repair has been the mainstay of treatment since the 1980's after Baker et al. (19) and Morrey et al. (18) published their research, stating that the power of supination decreased by $40 \%$ and endurance decreased by $47 \%$, with patients reported having trouble with tasks requiring supination. Since then the focus in the literature has been to look at the optimal surgical intervention for the best outcomes, until recently in 2007 where Chillemi et al. (20) compared surgical intervention to non-operative intervention. Even though they concluded that surgical intervention was superior it stimulated the question of what the outcomes were in those treated non-operatively. Since 2007 there have been numerous studies published looking into this. The majority have been retrospective in nature and with low numbers, which is expected given that surgical intervention has been the convention for decades (table II).

Chillemi et al. conducted a retrospective study looking at nine patients who had sustained traumatic total distal biceps brachii tendon ruptures, five of which underwent surgery, and four patients were treated conservatively. All the patients were male and had a median age of 49 . Patients were followed up after median 24 months. The SECEC elbow score was the only outcome measure used. Otherwise the patients were clinically and radiologically examined. They discovered a minimal deficit of flexion and extension in two patients, decreased supination in six patients and decreased pronation in four patients. Two patients experienced a postoperative paralysis of the deep branch of the radial nerve, which recovered after two and six months. However, they did not specify from which group these findings were found in. They noted that on those who had surgical intervention, two patients were reported to have radial tuberosity heterotopic bone formation at the site of tendon re-insertion, as well as ectopic ossification more proximally in the biceps muscle region. Looking at their SECEC results, each item tested was in favour of surgery and therefore they simply found that surgical management was superior to conservative management. Even though the paper did not explore the outcomes of the non-operative group in great detail, it is significant as it was one of the first papers in recent times to look into both management options (20).

Hetsroni et al. conducted a retrospective study which looked at 29 middle-aged men with a mean age of 47 who had been diagnosed with total distal biceps brachii tendon rupture.
Twelve of the patients were treated non-operatively, whereas seventeen were treated with surgical management. Patients functional outcome was evaluated using both the DASH and the modified Neer scale questionnaires. Isokinetic evaluation was also undertaken. In those operated on, the range of forearm rotation declined by a mean of 11 degrees in supination and 17 degrees in pronation compared to no deficit in both supination and pronation in the conservatively managed group. Functional outcome evaluation using both the DASH and Neer scale questionnaires demonstrated superior functional outcome and increased satisfaction in the surgically managed group compared to the conservatively managed group. A mean loss of $20 \%$ of the maximum strength of elbow flexors and a minimal flexor endurance deficit were observed for those treated conservatively when isokinetic testing was carried out. The operated group had regained full strength when tested. When testing supination, maximal strength was reduced by $35 \%$ and endurance reduced by $40 \%$ in those treated conservatively. Whereas in the operated group there was $15 \%$ loss in supination maximal strength and $10 \%$ supination endurance loss. Two patients treated with surgery suffered complications. One patient gradually developed median nerve paraesthesia and was noted to have heterotopic ossification of the proximal volar forearm after six months of the operation date. Symptoms were not spontaneously resolved, and the calcified mass was removed surgically, followed by full resolution of symptoms. The second case involved impaired posterior interosseous neuropathy, immediately after surgery and resolved four weeks later. This study made a point to exclude athletes and only included middle aged men as they believed this to be representative of the majority of patients who suffer this injury. They concluded that even though surgical repair of a completely avulsed distal biceps brachii tendon achieves a greater subjective functional outcome and greater strength and endurance. Patients should be made aware that non-operative management is likely to lead to good to excellent functional outcomes, avoiding possible surgical complications (21). The weakness of this paper was that it had a low number of patients.

Freeman et al. conducted a retrospective study comparing patients who had been treated non-operatively in their institute for total distal biceps brachii tendon rupture with a historical control using past studies in which patient's had been treated surgically. Twenty men and two women were identified in their institute who had been treated conservatively, of which four were excluded from the study. The median age was fifty years and the median follow up was thirty-eight months. Functional outcomes were measured using the MEPI, BMFI and DASH questionnaires. Isokinetic strength testing was also undertaken. The affected arm 


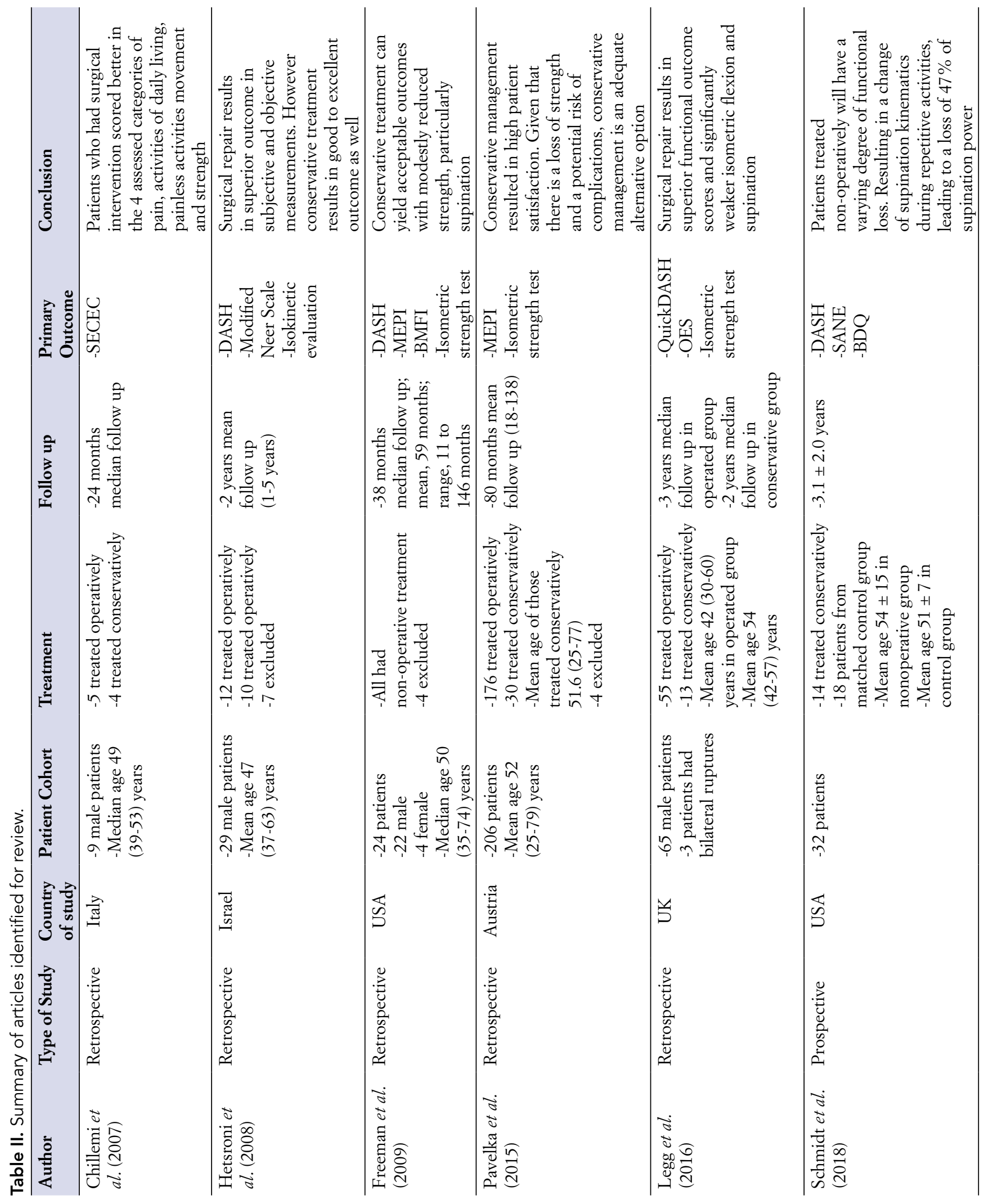


was found to have a median supination strength of $63 \%$ and a median flexion strength of $93 \%$ compared to the uninjured arm. In those who had suffered the injury in the non-dominant arm had good results when strength was compared to the uninjured dominant contralateral arm. The non-dominant arms had median flexion and supination strengths of $80 \%$ and $71 \%$, respectively, compared to the uninjured arm. The median flexion and supination strengths of those with injuries to the dominant arm were $63 \%$ and $100 \%$, respectively, compared to the uninjured arm. Every patient had a full range of motion, with the exception of one, who was noted to have an extension flexion arc of 10 degrees to 115 degrees, 80 degrees of supination and 70 degrees of supination. Looking at the functional outcome scores patients returned satisfactory scores overall. Eight patients described subjective weakness when attempting to lift weighty items and six patients described subjective weakness when supinating the affected arm. A significant difference in mean strength between the dominant affected arms and the non-dominant affected arms in terms of flexion were noted when statistically analysing these patients, but not in terms of supination. When comparing their patients to the historical control group the authors concluded that patients managed non-operatively achieved satisfactory results in both strength testing and functional outcome scores. Given the advantage of avoiding operational risk, their research suggested that those who are cautious about getting surgery, who present late or who are too unwell to undergo surgery, conservative management of distal biceps brachii tendon rupture is likely to accomplish acceptable results with modestly decreased supination strength (4). The patients in this study were compared to a historical control group derived from the literature and therefore leading to inaccuracies in comparing strength scores as there was a difference in equipment used when testing isokinetic strength. However, they did compare the injured arm to the uninjured arm and stated whether it was dominant or non-dominant hand when testing strength.

Pavelka et al. conducted a retrospective study looking at two hundred and six patients who had suffered from partial or total distal biceps brachii tendon rupture in their institute between 1999 and 2010. Of These patients, thirty were treated non-operatively. Functional outcomes were measure using MEPI and isokinetic strength measurements of supination and flexion were tested. Twenty-four patients were followed up, of which the mean age was 51 years. The group consisted of twenty-two men and two women. Of these patients, four were noted to have a partial rupture. In this study, it was shown that the mean Range of Motion (ROM) for flexion to be 139.1 degrees, for pronation to be 85 degrees and for supination to be 83.75 degrees. Elbow extension was normal in all patients except one who had a 20-degree deficit. All patients apart from two had normal supination. When examining the strength of the injured arm and comparing it with the un-injured arm, the loss of strength in the flexed position was $16.1 \%, 17.3 \%$ in the neutral position and $18.1 \%$ in the supinated position. A minor decrease in flexion strength was observed in the group of partial ruptures and no difference in supination strength was discovered. The authors found that their research indicates that we should also reconsider always opting for surgical management as the suggested treatment option can also produce excellent outcomes. Especially in the elderly and those who have low physical demands, non-operative management may be a suitable option. This study had a small number of patients treated non-operatively as well as including patients with partial ruptures of the distal biceps brachii tendon repair. They did not compare the group to those treated surgically and stated they were unable to perform statistical analysis due to the small numbers (22).

Legg et al. conducted a retrospective cohort study of patients who had suffered total biceps brachii tendon rupture and who had either surgical treatment or treated conservatively. 65 patients were identified from 2002 until 2013 and all were male. 55 patients underwent surgical treatment and 13 were treated conservatively. Of these patients 50 were available for follow up. The mean age of the operated group was 42 years compared to 54 years in the non-operated group. The average follow-up was 3 years in the operated group and 2 years in the non-operated group. Isokinetic strength was tested, and functional outcomes were measured using the OES, MEPI and QuickDASH questionnaires. 75\% of patients had ruptured their distal biceps brachii tendon in their dominant hand. Functional outcome questionnaires revealed significantly better mean scores in the operated group. In either cohort, the range of motion was not significantly reduced compared to the uninjured arm. The isometric flexion and supination strength differed significantly when the operated group was compared with the conservatively managed group, the latter being weaker. Two patient's developed posterior interosseous neuropath in the surgically treated group, which recovered completely within 3 months. Incidental heterotopic ossification and tunnel lysis was noted in these patients but no impact on function was found. The authors concluded that surgical management restored normal flexion and supination as well as near normal strength in these planes of motion. In doing so, they did not focus on the fact that their results showed acceptable outcomes of those treated conservatively.

Schmidt et al. undertook a prospective cross-sectional study in their institution comparing nonoperatively managed patients who had unilateral complete distal biceps brachii 
tendon rupture with a matched unaffected control group. The functional outcomes were measured using the DASH, SANE and BDQ questionnaires. Isometric testing was undertaken using a custom device designed to re-produce high intensity forearm supination motions. 14 patients who were all male, had an average age of 54 and had sustained a distal biceps brachii tendon rupture participated in the study. The time between injury and testing was 3.1 years. 12 of the men injured their dominant arm. The non-operative patients reported having difficulty in light or intense tasks or both. The functional outcome scores in the control group were significantly better compared to the non-operated group. It was observed that non-operatively managed patients replaced the lost biceps function by increasing the adduction of the shoulder to perform supination activities. On average, these patients reduced their arc of supination by $27 \%$, transferring the centre of the rotation arc to 45 degrees in a more pronated position and using $27 \%$ more shoulder adduction to perform the tests. The study also was able to show a power reduction of up to $47 \%$ in the injured arm. The authors found that the non-operated patients perceive some degree of impairment and that the patients compensated by locking their wrist and adducting their shoulder to perform supination tasks. This study is the first to state that there is a greater loss in elbow supination rather than elbow flexion as noted in all the other studies. The comparison in this study was made with a control group made up of uninjured patients and therefore potentially exaggerating the differences noted between the two groups. Surgical management has been the mainstay of treatment for decades and has been considered to result in considerably greater outcomes, resulting in the lack of high-level studies to evaluate this treatment method. This has led to the majority of studies comparing surgical methods without considering the outcome of those treated non-operatively.

\section{Limitations}

This systematic review focused on 5 retrospective studies and 1 prospective study. There were no randomised controlled trials conducted in this area. Therefore, high quality evidence is lacking from this review.

\section{REFERENCES}

1. Khan W, Agarwal M, Funk L. Repair of distal biceps tendon rupture with the Biotenodesis screw. Arch Orthop Trauma Surg 2004;124(3):206-8.

2. Mazzocca, AD, Alberta FG, Elattrache NS, Romeo AA. Single incision technique using an interference screw for the repair
An extensive search was performed, however there is a possibility that studies eligible for review were not identified due to a possible lack of robustness in the search terms or due to publication bias. A language bias was also introduced as studies written in languages other than English were excluded. Study selection and data extraction was carried out by one reviewer, introducing a selection bias. The literature says that at least two assessors are needed to evaluate data and conduct analyses to prevent selection bias (26). Regarding the studies identified there was range of questionnaires used to assess functional outcome. When undertaking isokinetic tests, there was a range of devices used to measure strength. The studies all had very low patient numbers.

One study included both total and partial ruptures. Two studies grouped male and female patients together.

\section{CONCLUSIONS}

This review highlights that the current evidence for surgical management of distal biceps brachii tendon rupture is weak. Studies in recent times have mostly focused on the different methods of surgical fixation. We noted that surgical fixation resulted in a large number of complications, which if communicated to the patient may result in them opting for conservative management.

However equally important to highlight is that patients who are middle-aged, with a modest physical demand should be made aware that if treated non-operatively they may likely have good to excellent functional outcome as well as having approximately $50 \%$ flexion and supination strength in the affected arm after injury, whilst avoiding the potential complications of surgery.

This review highlights the need for higher level evidence. Given that the outcomes of the non-operatively treated patients are satisfactory, this review challenges the status quo and advocates that higher-level studies should be conducted. Non-operative treatment can be a good alternative in those who are less active and of low demand.

\section{CONFLICT OF INTERESTS}

The authors declare that they have no conflict of interests.

of distal biceps tendon ruptures. Oper Tech Sports Med 2003;11(1):36:41.

3. Agins HJ, Chess JL, Hoekstra DV, Teitge RA. Rupture of the distal insertion of the biceps brachii tendon. Clin Orthop Relat Res 1988;(234):34-8. 
4. Freeman CR, McCormick KR, Mahoney D, Baratz M, Lubahn JD. Nonoperative treatment of distal biceps tendon ruptures compared with a historical control group. J Bone Joint Surg Am 2009;91(10):2329-34.

5. Safran MR, Graham SM. Distal biceps tendon ruptures: incidence, demographics, and the effect of smoking. Clin Orthop Relat Res 2002;(404):275-83.

6. Leighton MM, Bush-Joseph CA, Bach BR Jr. Distal biceps brachii repair. Results in dominant and nondominant extremities. Clin Orthop Relat Res 1995;(317):114-21.

7. Bisson L, Moyer M, Lanighan K, Marzo J. Complications associated with repair of a distal biceps rupture using the modified two-incision technique. J Shoulder Elbow Surg 2008;17(1 Suppl):67S-71S.

8. Kelly EW, Morrey BF, O'Driscoll SW. Complications of repair of the distal biceps tendon with the modified two-incision technique. J Bone Joint Surg Am 2000;82(11):1575-81.

9. Eames MH, Bain GI, Fogg QA, van Riet RP. Distal biceps tendon anatomy: a cadaveric study. J Bone Joint Surg Am 2007;89(5):1044-9.

10. Dobbie, R. P. Avulsion of the lower biceps brachii tendon. Analysis of fifty-one previously unreported cases. Am J Surg 1941;51(3):662-683.

11. Guglielmino C, Massimino P, Ioppolo F, Castorina S, Musumeci G, Di Giunta A. Single and dual incision technique for acute distal biceps rupture: clinical and functional outcomes. MLTJ 2016;6(4):453-460.

12. Boyd, HB, Anderson, LD. A Method for Reinsertion of the Distal Biceps Brachii Tendon. J Bone Jt Surg 1961;43(7):1041-1043.

13. Rollo G, Meccariello L, Rotini R, Pichierri P, Bisaccia M, Fortina M. Efficacy of the "Salento technique", a modified two-incision approach in distal biceps brachii tendon repair. Surgical description and outcomes analysis. J Clin Orthop Trauma 2019;10(5):959-964.

14. Bell RH, Wiley WB, Noble JS, Kuczynski DJ. Repair of distal biceps brachii tendon ruptures. J Shoulder Elbow Surg 2000;9(3):223-6.

15. Lanzetti, RM, Lupariello D, Topa D, Spoliti M. Reconstruction of acute distal biceps tendon ruptures with ToggleLoc. Clini- cal, functional and radiographic outcomes at 5-year follow-up. MLTJ 2019;9(2):241-245.

16. Cohen MS. Complications of distal biceps tendon repairs. Sports Med Arthrosc Rev 2008;16(3):148-53.

17. Carroll R, Hamilton L. Rupture of biceps brachii-a conservative method of treatment. Proc Am Acad Orthop Surg J Bone Jt Surg Am 1967:49.

18. Morrey BF, Askew LJ, An KN, Dobyns JH. Rupture of the distal tendon of the biceps brachii. A biomechanical study. J Bone Joint Surg Am 1985;67(3):418-21.

19. Baker BE, Bierwagen D. Rupture of the distal tendon of the biceps brachii. Operative versus non-operative treatment. J Bone Joint Surg Am 1985;67(3):414-7.

20. Chillemi C, Marinelli M, De Cupis V. Rupture of the distal biceps brachii tendon: conservative treatment versus anatomic reinsertion--clinical and radiological evaluation after 2 years. Arch Orthop Trauma Surg 2007;127(8):705-8.

21. Hetsroni I, Pilz-Burstein R, Nyska M, Back Z, Barchilon V, Mann G. Avulsion of the distal biceps brachii tendon in middle-aged population: is surgical repair advisable? A comparative study of 22 patients treated with either nonoperative management or early anatomical repair. Injury 2008;39(7):753-60.

22. Pavelka M, Pienaar S, Fialka C, Obrist J Distal biceps tendon rupture - Is surgical reconstruction really always necessary? Sport Orthop Traumatol 2015;31:267-271.

23. PRISMA. PRISMA - Transparent Reporting of Systematic Reviews and Meta-analyses. PRISMA 2015.

24. Riva JJ, Malik KM, Burnie SJ, Endicott AR, Busse JW. What is your research question? An introduction to the PICOT format for clinicians. J Can Chiropr Assoc 2012;56(3):167-71.

25. CASP. Critical Appraisal Skills Programme (CASP). Qualitative Checklist 2018.

26. Higgins J, Green S (eds). Cochrane Handbook for Systematic Reviews of Interventions Version 5.1.0. Cochrane Collaboration, 2011.

27. Padulo J, Oliva F, Frizziero A, Maffulli N. Muscles, Ligaments and Tendons Journal - Basic principles and recommendations in clinical and field Science Research: 2016 Update. MLTJ 2016;6(1):1-5. 